\title{
Beiträge zur Kenntnis der Radioaktivität der Mineralquellen Tirols
}

\author{
(V. Mitteilung) \\ von \\ Max Bamberger und Karl Krüse.
}

Aus dem Laboratorium für anorganische Chemie an der k. k. Technischen Hochschule in Wien und aus dem Physikalischen Kabinett des k. k. Reformrealgymnasiums in Bozen.

(Mit 1 Textfigur.)

(Vorgelegt in der Sitzung am 8. Mai 1913.)

Im nachstehenden finden sich die Resultate der im Jahre 1912 ausgeführten Arbeiten über die Radioaktivität der Mineralquellen Tirols zusammengestellt.

Zur Bestimmung der Radioaktivität dienten das Engler sche Fontaktoskop ${ }^{2}$ sowie das Fontaktometer ${ }^{3}$ von Mache und Meyer.

Die in den Tabellen angegebenen Zahlen geben den für $1 l$ Wasser direkt ermittelten oder, da in Fällen geringere Wassermassen benutzt wurden, den für $1 l$ umgerechneten Potentialabfall in Volt pro 1 Stunde unter Berücksichtigung des Normalverlustes, der induzierten Aktivität sowie der im Versuchswasser zurückgebliebenen Emanation an.

1 Monatshefte für Chemie, 29, 317 (1908); 31, 221 (1910); 32, 797 (1911); 34, 403 (1913).

2 Kapazität der Elektroskope Nr. 2220 und 2211: $13 \cdot 9$ und 13.4.

3 Kapazität des Elektroskops Nr. 2733 betrug $10 \cdot 8$. 


\begin{tabular}{|c|c|c|c|c|c|c|c|c|c|c|c|}
\hline 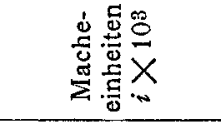 & & $\begin{array}{l}8 \\
\dot{n}\end{array}$ & 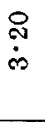 & $\begin{array}{l}\stackrel{\infty}{O} \\
\stackrel{-}{-}\end{array}$ & $\begin{array}{l}\stackrel{\infty}{d} \\
\dot{n}\end{array}$ & $\stackrel{=}{\rightleftarrows}$ & $\begin{array}{l}\text { : } \\
\text { is }\end{array}$ & $\begin{array}{l}\stackrel{y}{+} \\
\dot{c}\end{array}$ & $\dot{0}$ & $\begin{array}{l}\mathscr{D} \\
\stackrel{8}{0} \\
\dot{N}\end{array}$ & $\dot{0}$ \\
\hline 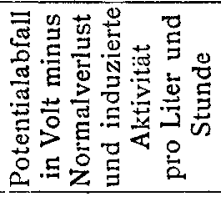 & & $\stackrel{\check{O}}{\dot{8}}$ & $\begin{array}{l}\dot{0} \\
\dot{g} \\
\stackrel{\sim}{+}\end{array}$ & $\begin{array}{l}\dot{0} \\
\dot{\infty}\end{array}$ & $\begin{array}{l}\stackrel{0}{\circ} \\
\dot{\sigma} \\
\stackrel{N}{S}\end{array}$ & $\begin{array}{l}\dot{0} \\
\dot{\infty} \\
\dot{\infty}\end{array}$ & $\begin{array}{l}O \\
\stackrel{Z}{Z} \\
\exists\end{array}$ & $\begin{array}{l}\stackrel{0}{\infty} \\
\dot{\infty} \\
\stackrel{\infty}{-}\end{array}$ & $\dot{\theta}$ & 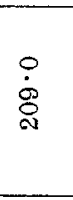 & $\dot{0}$ \\
\hline 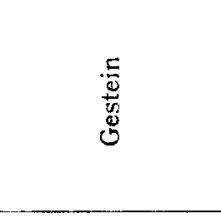 & & 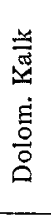 & * & A & 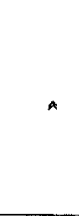 & 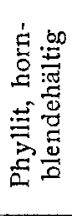 & 密 & $\frac{\sqrt[N]{3}}{\partial}$ & $\frac{n}{0}$ & 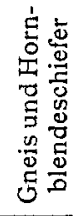 & 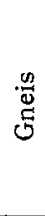 \\
\hline 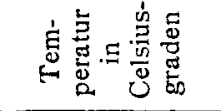 & & $\begin{array}{l}0 \\
\dot{\infty}\end{array}$ & $\stackrel{10}{\infty}$ & $\stackrel{\infty}{\infty}$ & $\dot{P}$ & $\dot{0}$ & $\dot{\infty}$ & $\dot{\phi}$ & 1 & $\stackrel{0}{\infty}$ & $\stackrel{\circ}{\dot{O}}$ \\
\hline 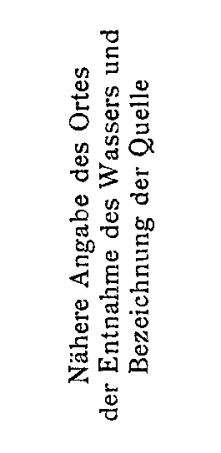 & & 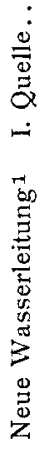 & $\begin{array}{l}: \\
. \\
\dot{\Xi} \\
\dot{\Xi}\end{array}$ & $\begin{array}{l}: \\
\stackrel{-}{\infty} \\
\dot{\infty}\end{array}$ & 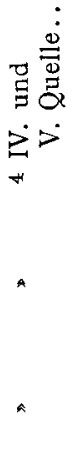 & 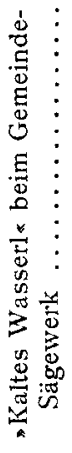 & 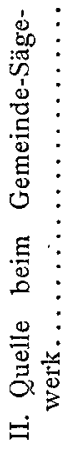 & 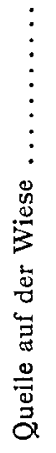 & 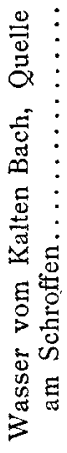 & 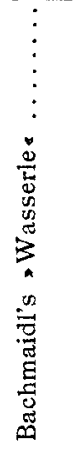 & $\begin{array}{c}\vdots \\
\vdots \\
\vdots \\
\vdots \\
\vdots \\
\vdots \\
0 \\
\vdots \\
0 \\
0 \\
0 \\
0 \\
0 \\
0 \\
0 \\
0 \\
0 \\
0\end{array}$ \\
\hline 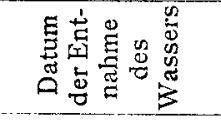 & $\frac{\sigma}{\sigma}$ & $\begin{array}{l}\text { 点 } \\
\stackrel{2}{\leftrightarrows}\end{array}$ & $\stackrel{a}{\check{E}}$ & $\stackrel{a}{=}$ & ^ & $\stackrel{\circ}{\circ}$ & $\stackrel{a}{\stackrel{0}{*}}$ & $\stackrel{a}{\circ}$ & $\stackrel{\mathscr{N}}{\stackrel{\oplus}{*}}$ & $\stackrel{\leftrightarrow}{\infty}$ & $\stackrel{0}{\circ}$ \\
\hline \multirow[b]{2}{*}{ 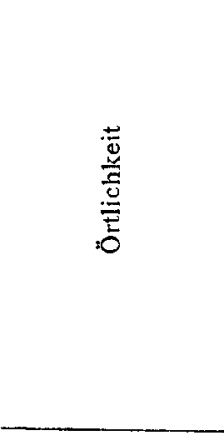 } & & 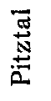 & A & a & a & $\wedge$ & $\wedge$ & a & ^ & A & A \\
\hline & & $\begin{array}{l}\bar{N} \\
\frac{5}{4}\end{array}$ & $\wedge$ & A & A & $\stackrel{5}{\stackrel{0}{0}}$ & * & 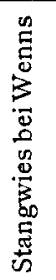 & $\frac{\mathrm{d}}{\stackrel{2}{2}}$ & $\frac{\vec{d}}{\vec{\omega}}$ & ^ \\
\hline
\end{tabular}




\section{另}

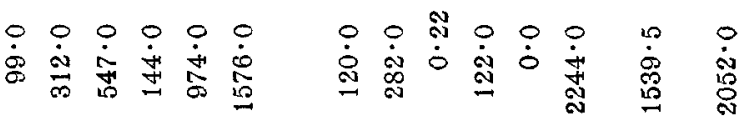

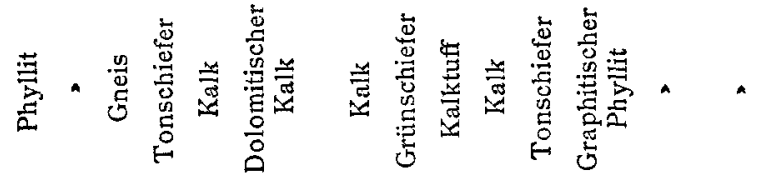

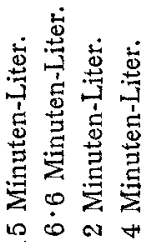

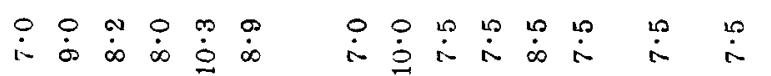

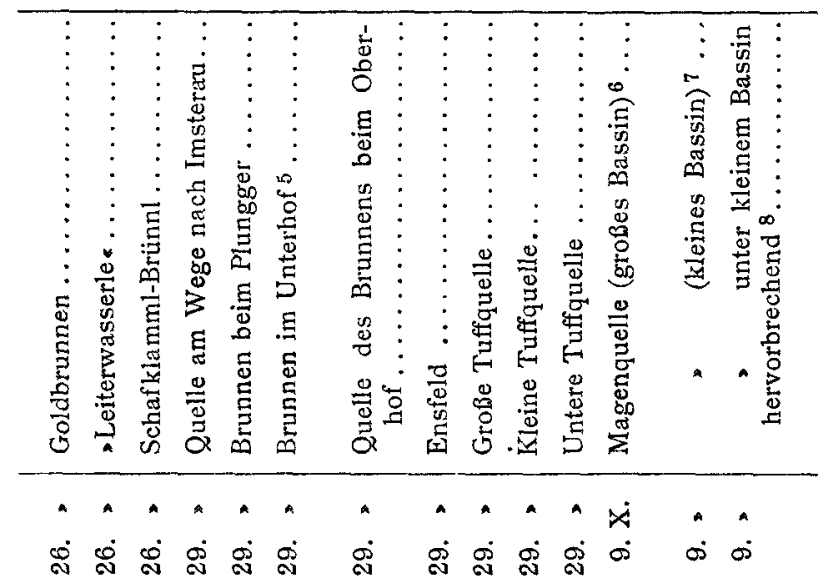

若节苛

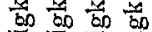
远

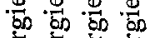
由于

$\infty \infty-\infty$

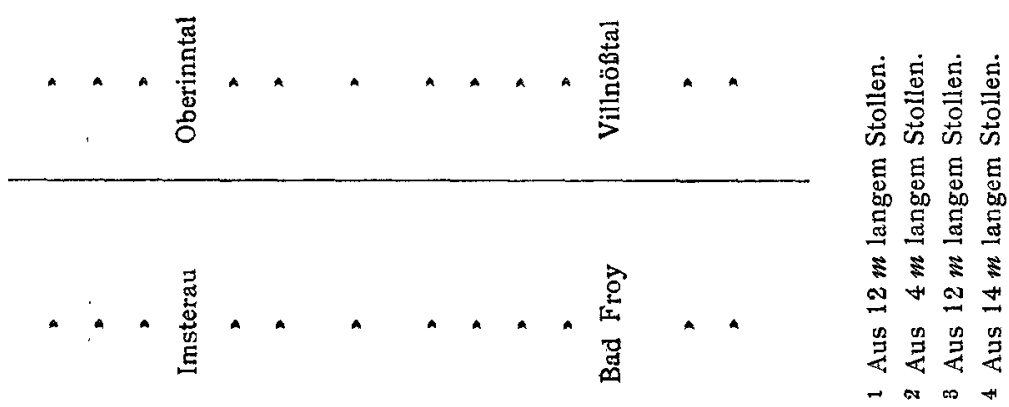


M. Bamberger und K. Krüse,

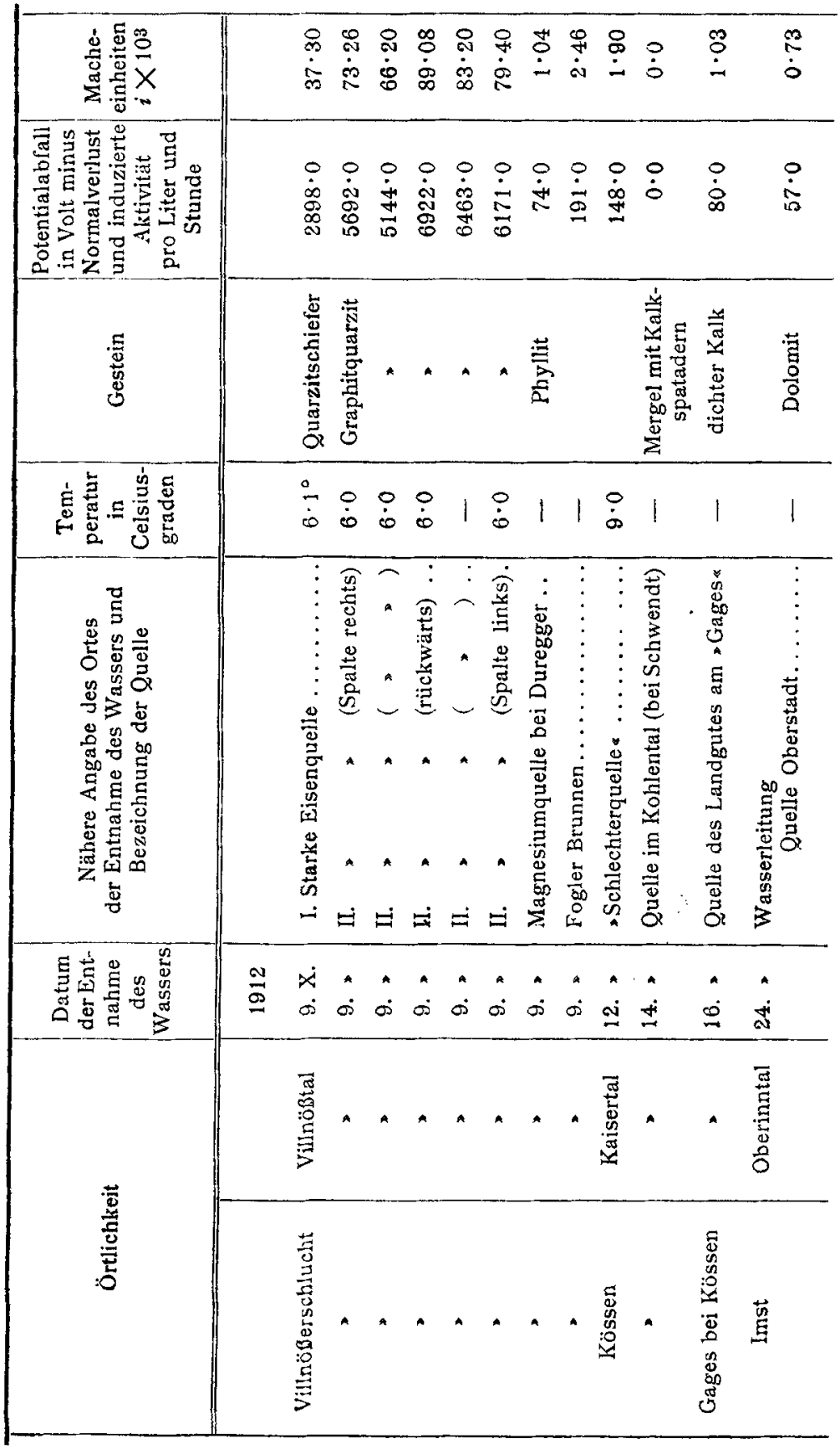




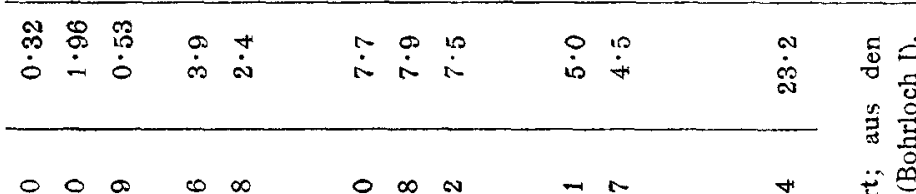

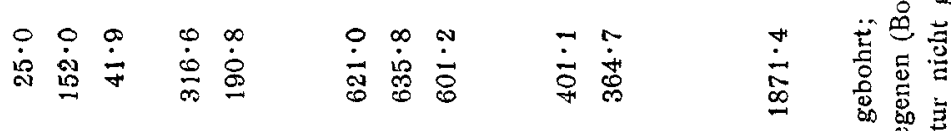

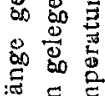

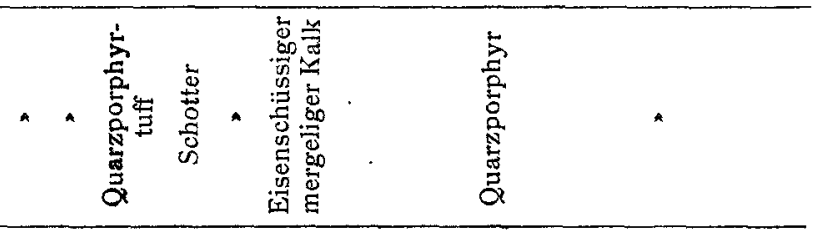

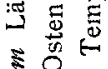

$\infty$ 过

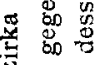

5 站 要

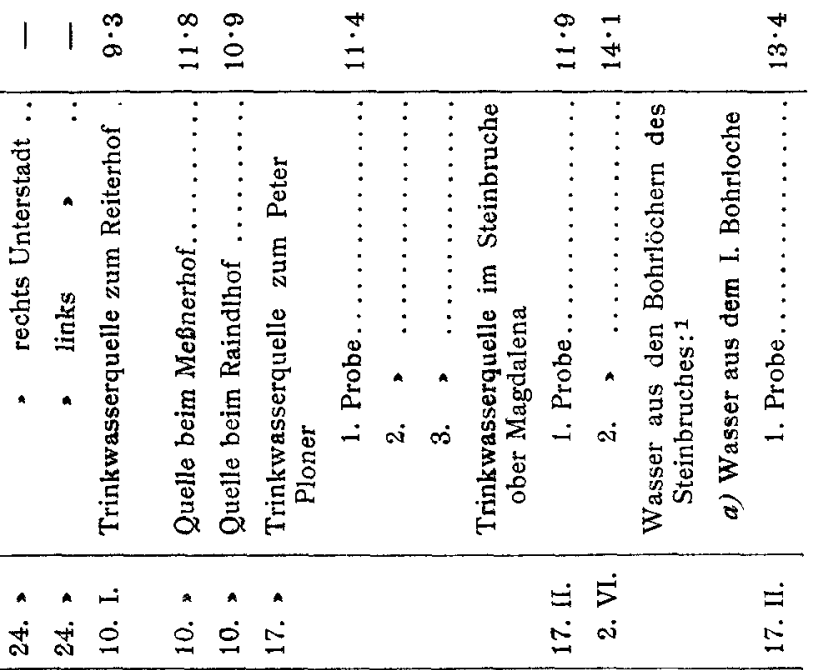

$>\stackrel{D}{=}$

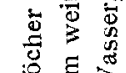
:

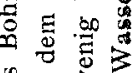
$\stackrel{5}{2} 3$ 造

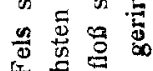
㽞 ․ㅓㄴ

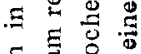
동 통으 声言 跑 品焉

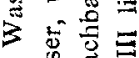

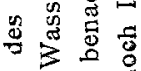
苛要

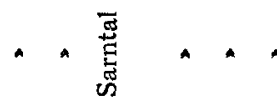

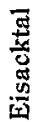

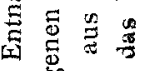
迹递总 熯吉竞

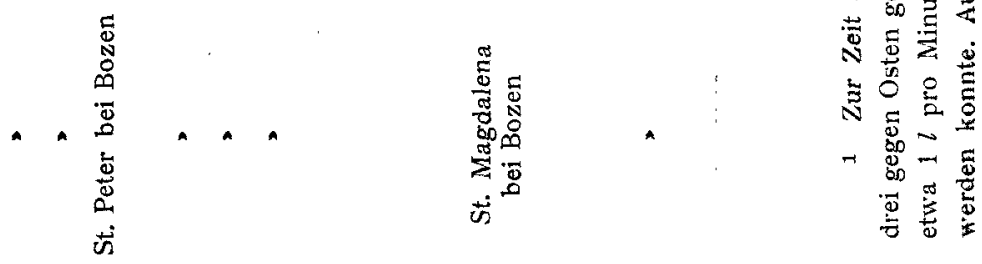


M. Bamberger und $\mathrm{K}$. Krüse,

\begin{tabular}{|c|c|c|c|c|c|c|}
\hline 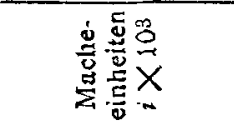 & 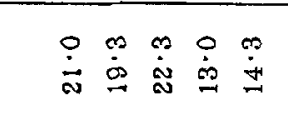 & 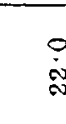 & 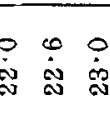 & & : & $\begin{array}{l}\stackrel{H}{+} \\
\dot{0}\end{array}$ \\
\hline 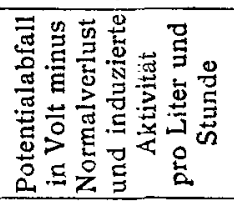 & 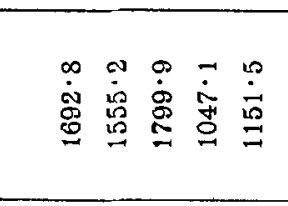 & 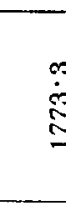 & 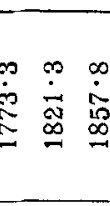 & & 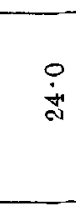 & $\ddot{\infty}$ \\
\hline 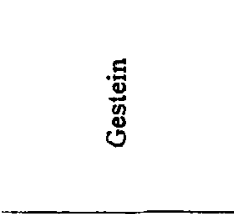 & 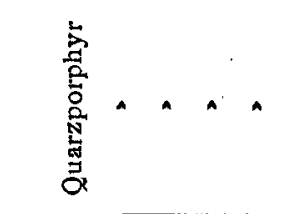 & a & & & $\begin{array}{l}\frac{D}{0} \\
0 \\
0 \\
0 \\
0 \\
0 \\
0 \\
0 \\
0 \\
0 \\
0\end{array}$ & 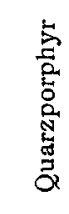 \\
\hline 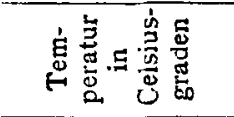 & 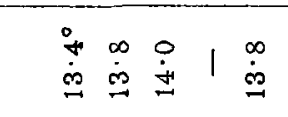 & & & & $\underset{\infty}{\infty}$ & $\stackrel{N}{\infty}$ \\
\hline 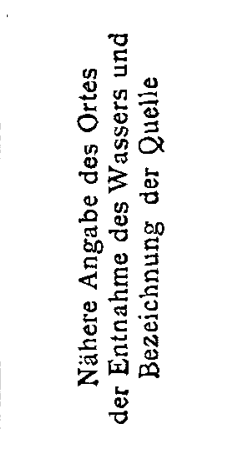 & 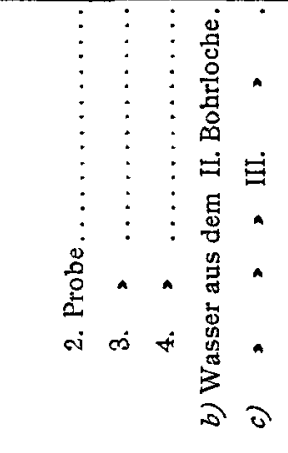 & 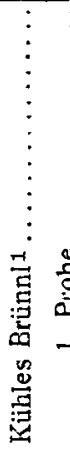 & 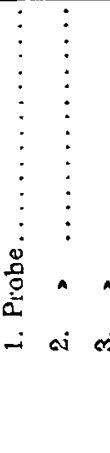 & 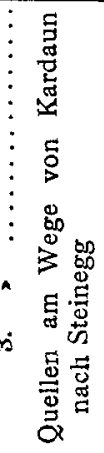 & 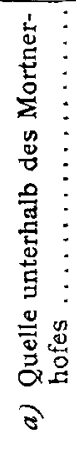 & 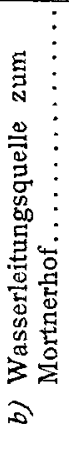 \\
\hline 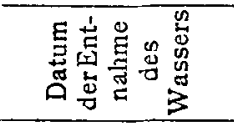 & 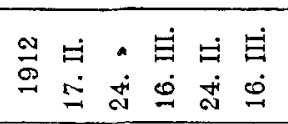 & $\begin{array}{l}\stackrel{3}{5} \\
\dot{\Xi}\end{array}$ & . & $\stackrel{\circ}{\circ}$ & & \\
\hline \multirow[b]{2}{*}{ 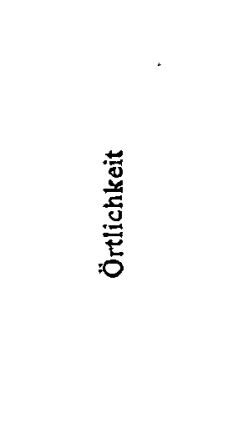 } & & 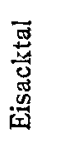 & & a & & \\
\hline & & 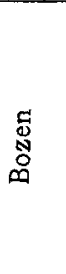 & & 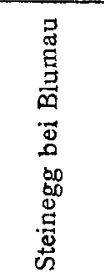 & & \\
\hline
\end{tabular}




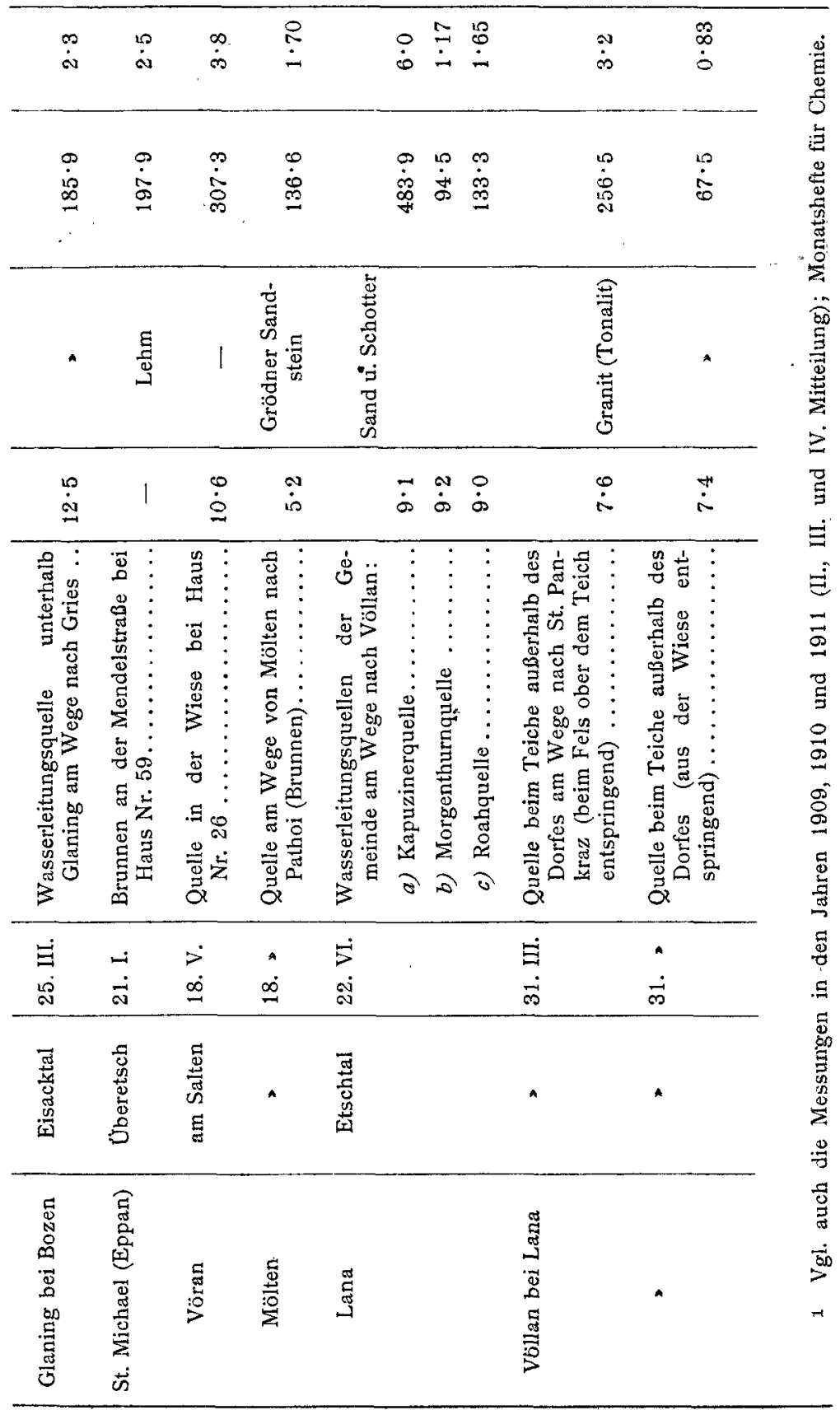


M. Bamberger und K. Krüse,

\begin{tabular}{|c|c|c|c|c|c|}
\hline 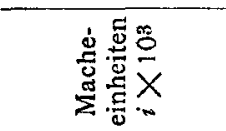 & & $\stackrel{\infty}{\dot{\alpha}} \overrightarrow{\dot{0}}$ & $\stackrel{+}{\Xi}$ & $\dot{\Delta} \stackrel{+}{\infty}$ & $\stackrel{\leftrightarrow}{\dot{p}}$ \\
\hline 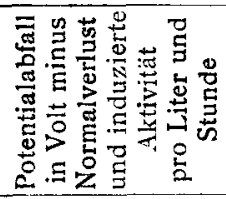 & & 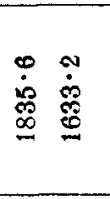 & $\frac{\dot{\leftrightarrow}}{a}$ & 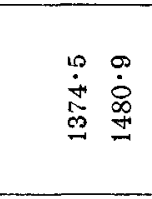 & 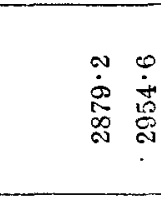 \\
\hline $\begin{array}{l}.5 \\
\mathbb{0} \\
0 \\
0 \\
0\end{array}$ & 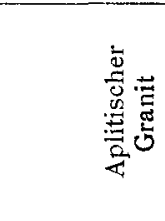 & & • & $A$ & ^ \\
\hline 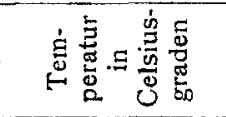 & & $\stackrel{0}{\stackrel{i}{i}} \dot{\infty}$ & $\stackrel{N}{i n}$ & $\dot{0}$ & $\stackrel{\infty}{\dot{\omega}}$ \\
\hline 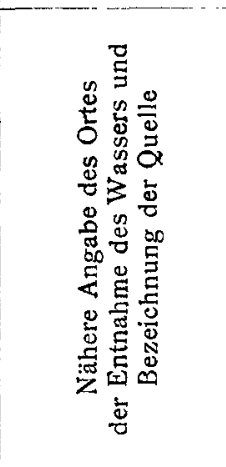 & 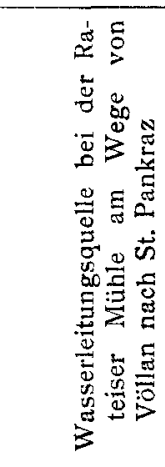 & $\begin{array}{cc} & \vdots \\
\vdots & \vdots \\
\vdots & \vdots \\
\vdots & \vdots \\
\vdots & \vdots \\
\vdots & \vdots \\
0 & \\
0 & \\
0 & \\
-i & \text { oi } \\
& \end{array}$ & 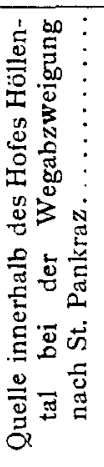 & 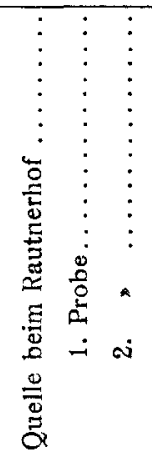 & 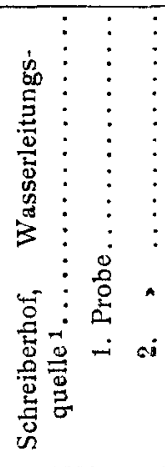 \\
\hline 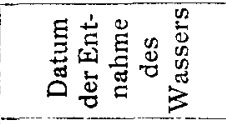 & $\stackrel{9}{\sigma}$ & $\begin{array}{l}\dot{\Xi} \\
\dot{5} \\
\dot{\infty}\end{array}$ & $\begin{array}{l}\dot{\Xi} \\
\dot{\infty}\end{array}$ & $\dot{Z}$ & $\begin{array}{l}\dot{5} \\
\text { త্ } \\
\end{array}$ \\
\hline \multirow[b]{2}{*}{ 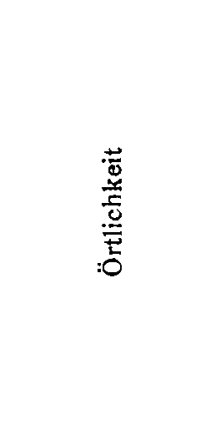 } & 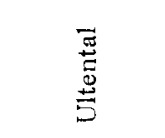 & & . & 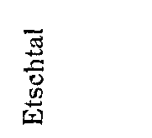 & $\star$ \\
\hline & 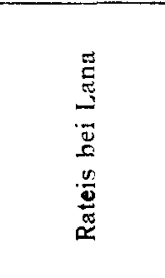 & & . & 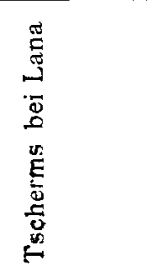 & ^ \\
\hline
\end{tabular}




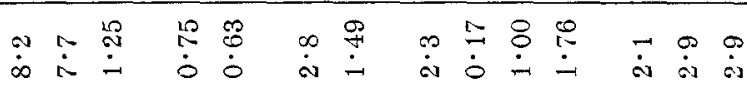

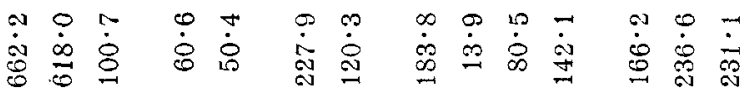

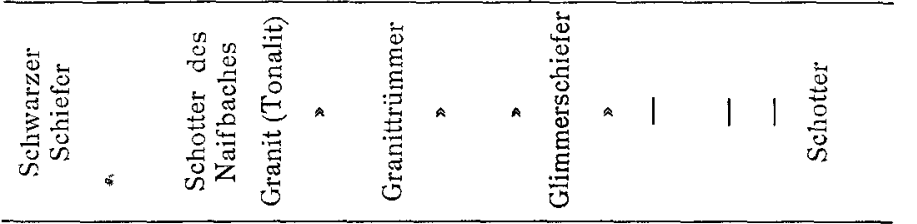

\begin{tabular}{|c|c|c|c|c|c|c|c|c|c|c|c|c|c|}
\hline$\stackrel{0}{\dot{\sim}}$ & & $\stackrel{15}{\dot{\alpha}}$ & $\dot{\omega}$ & $\dot{p}$ & $\ddot{0}$ & $\begin{array}{l}\dot{0} \\
\dot{0}\end{array}$ & $\ddot{0}$ & $\stackrel{\sim}{\check{\beth}}$ & $\begin{array}{l}+ \\
\dot{a}\end{array}$ & | & 1 & & $\dot{\phi}$ \\
\hline 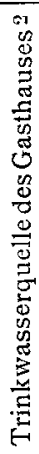 & $\begin{array}{c}0 \\
\vdots \\
\vdots \\
\vdots \\
\vdots \\
\vdots \\
0 \\
0 \\
0 \\
0 \\
-\end{array}$ & 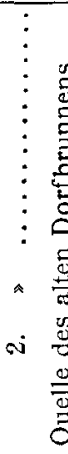 & 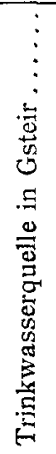 & 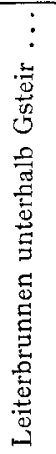 & 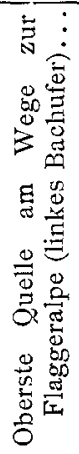 & 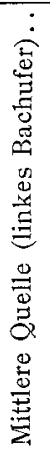 & 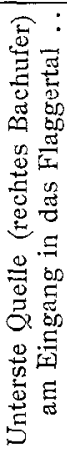 & $\begin{array}{c}\vdots \\
\vdots \\
\vdots \\
\vdots \\
\dot{0} \\
0 \\
0 \\
0 \\
0 \\
0 \\
0 \\
0 \\
0 \\
0 \\
0 \\
0 \\
0 \\
0 \\
0 \\
0 \\
0\end{array}$ & 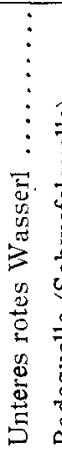 & 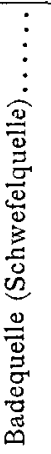 & 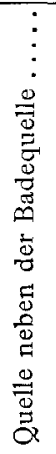 & 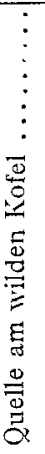 & 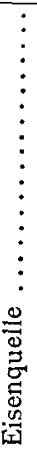 \\
\hline $\overrightarrow{>}$ & & 7 & 5 & 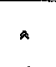 & $>$ & a & . & $\Xi$ & a & * & A & " & A \\
\hline$\dot{\varphi}$ & & 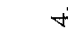 & $\sigma^{\circ}$ & $\dot{0}$ & $\stackrel{\mathrm{N}}{\mathrm{N}}$ & ลิ & ่ㅓํ & $\stackrel{\infty}{\check{0}}$ & $\stackrel{\circ}{\Omega}$ & & 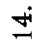 & $\stackrel{\leftrightarrow}{\leftrightarrows}$ & $\stackrel{10}{\Omega}$ \\
\hline
\end{tabular}




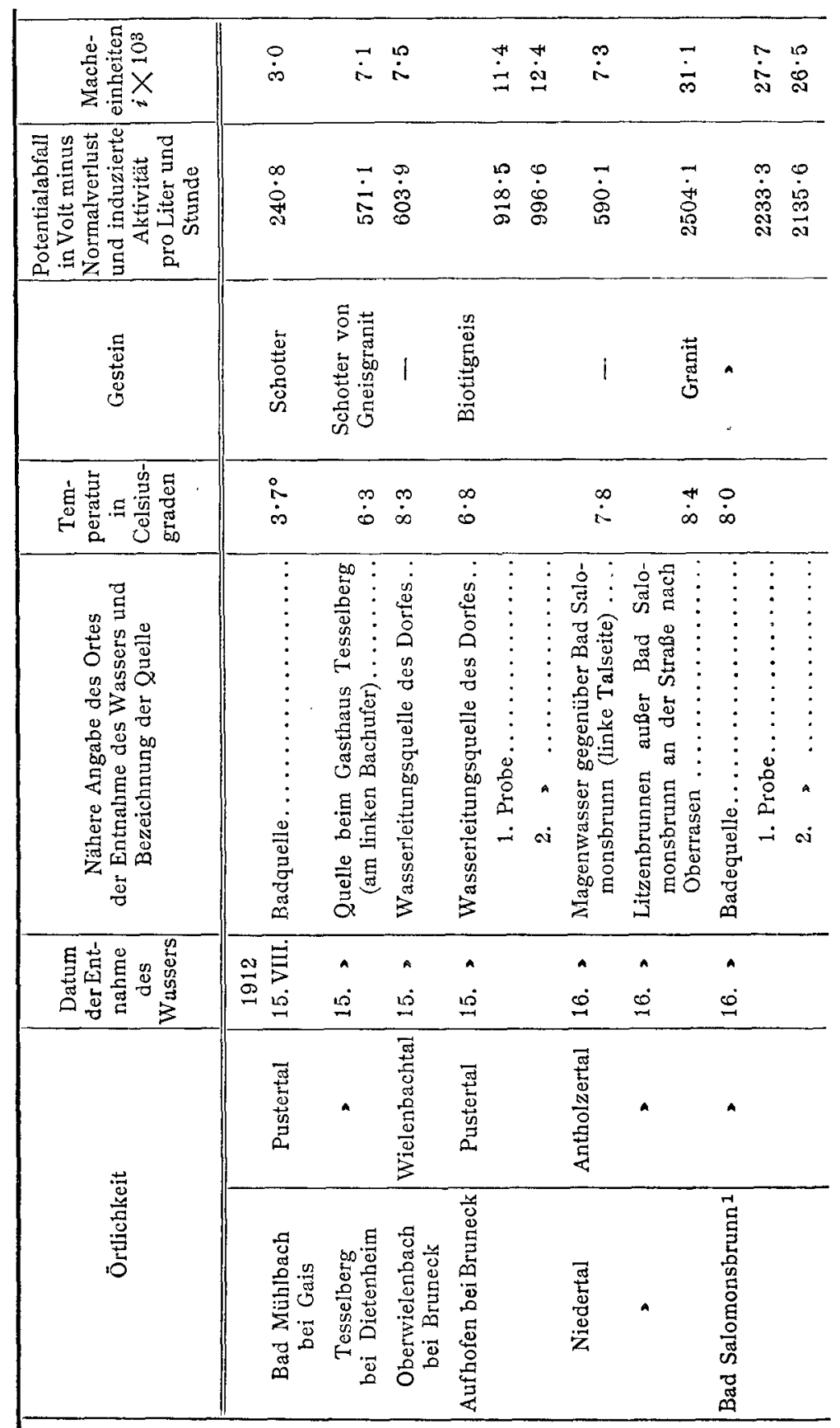


Radioaktivität der Mineralquellen Tỉrols.

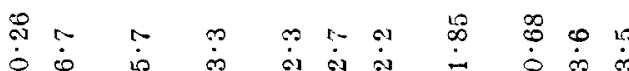

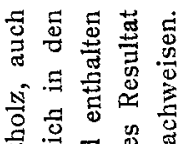

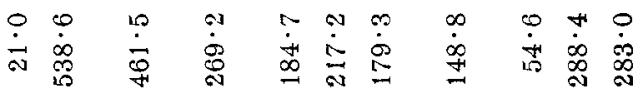

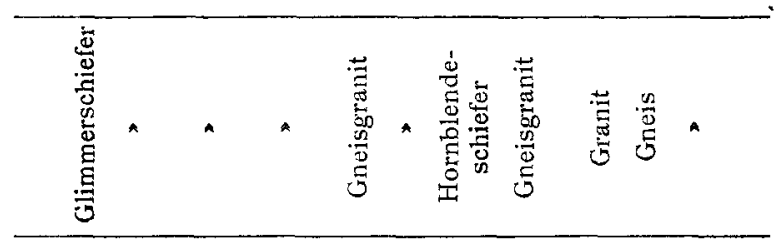

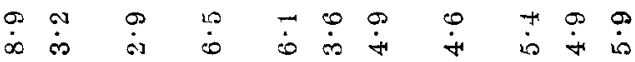

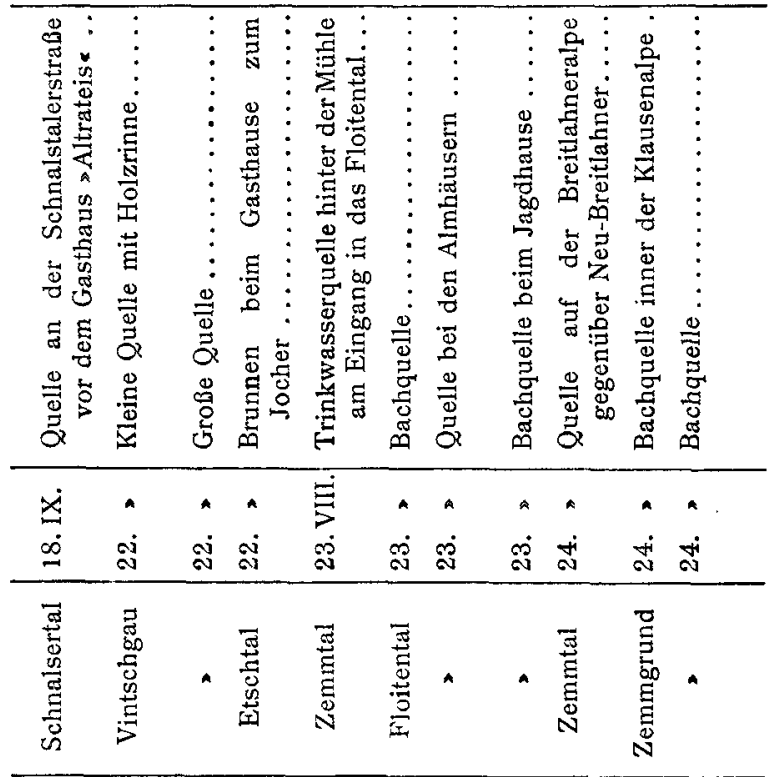

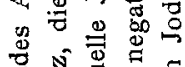

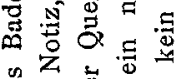

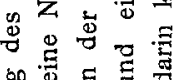

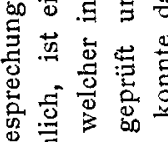

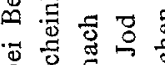
s.

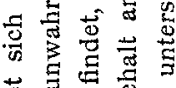
똘 要

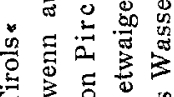

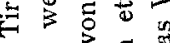
है

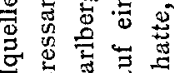
焉焉

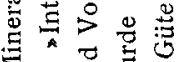

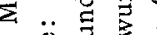
虽总 $\overrightarrow{0}$

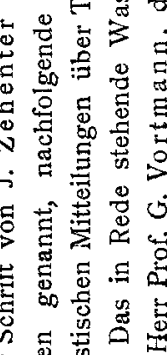

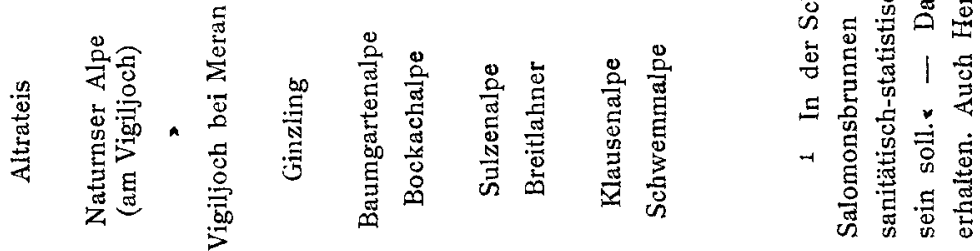


M. Bamberger und K. Krüse,

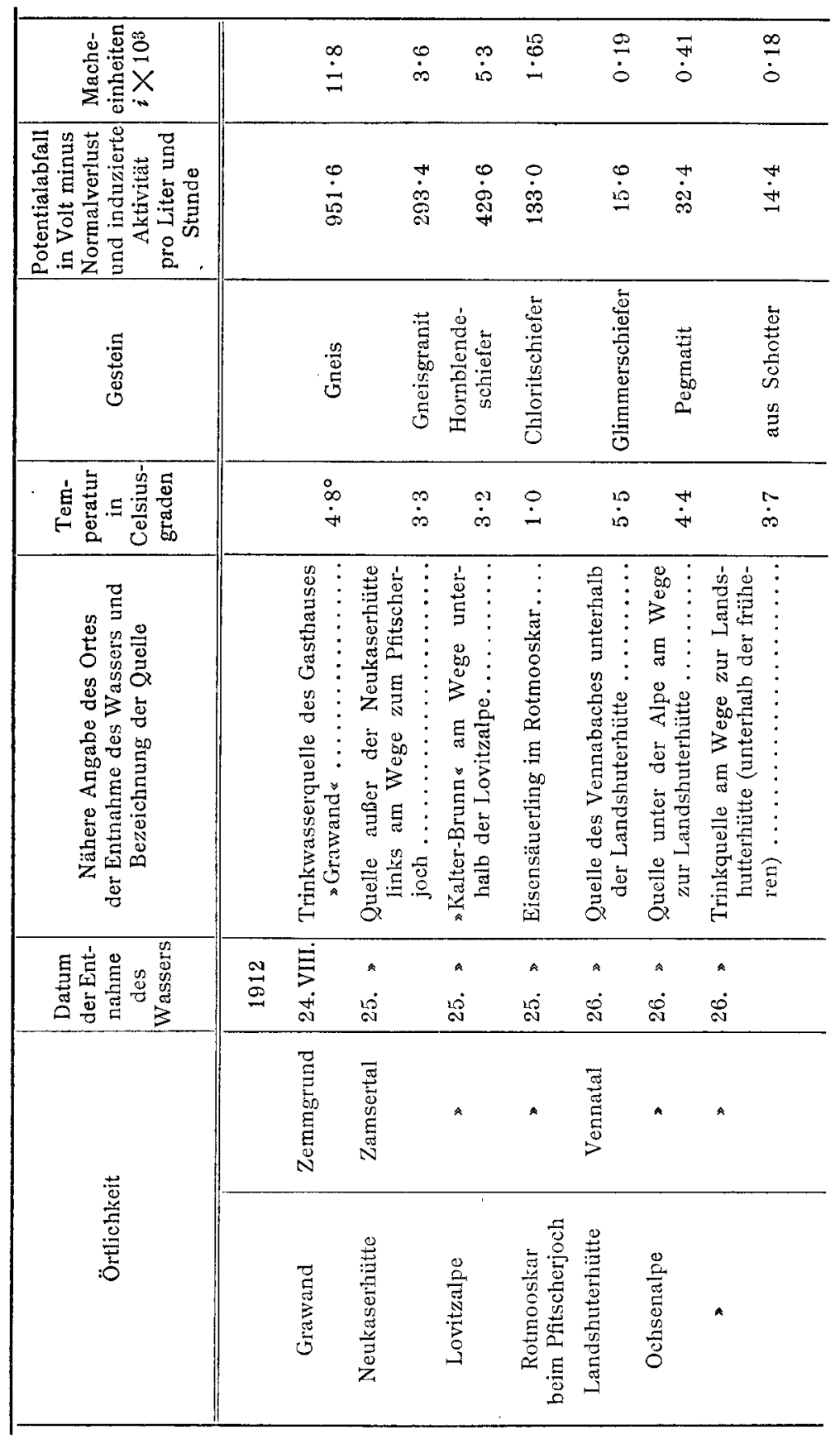


Radioaktivität der Mineralquellen Tirols.

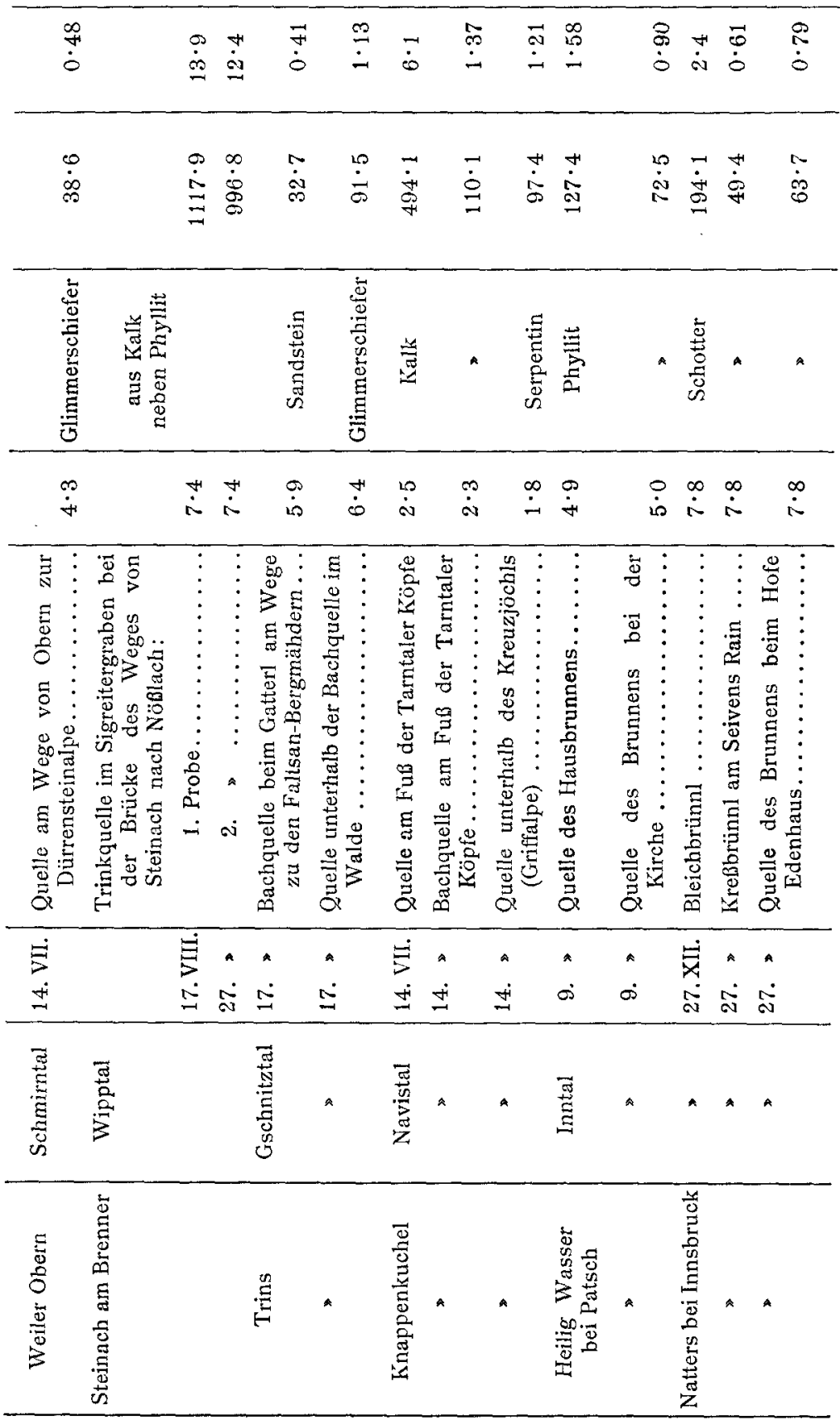


M. Bamberger und K. Krüse,

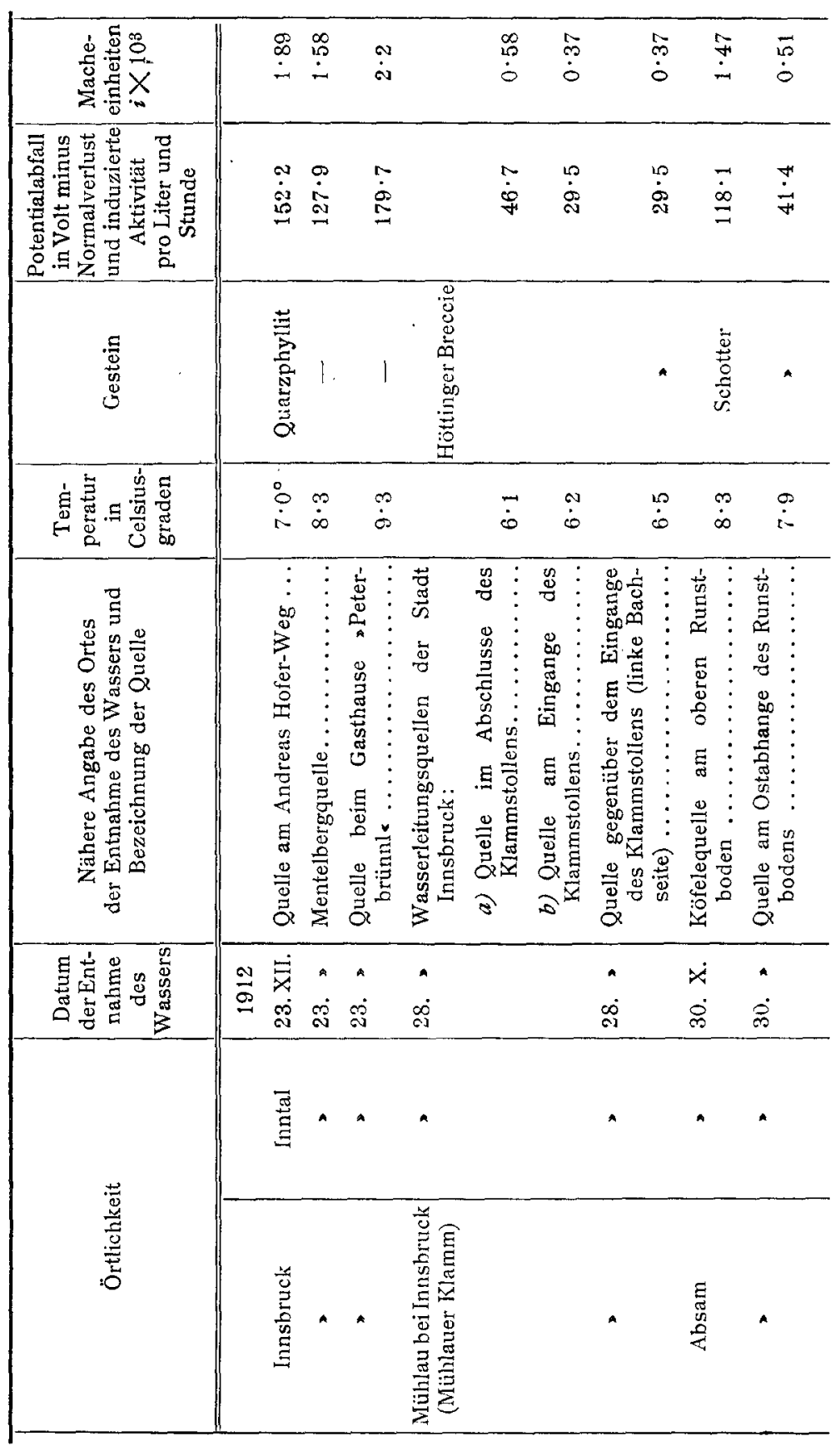


Einige der stärker radioaktiven Wässer (Quellen beim Schreiberhof in Tscherms, beim Rautnerhof in Tscherms, bei der Rateiser Mühle, Kühles Brünnl [Bozen], Quelle im Steinbruche bei St. Magdalena [Bozen], Quelle beim Peter Ploner [Bozen]) wurden auf ihren Gehalt an Restaktivität geprüft und keine nennenswerten Beträge für dieselbe erhalten.

Die Abklingung der induzierten Aktivität des Wassers aus dem Steinbruch in St. Magdalena bei Bozen zeigt folgende Figur.

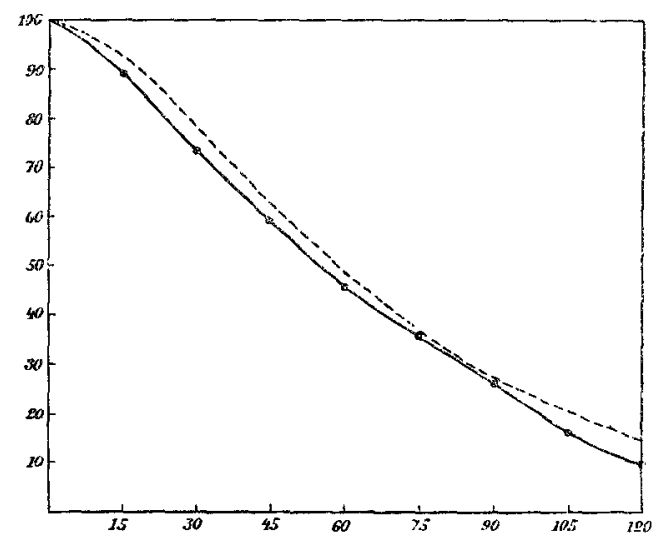

- - - _ Radium nach Curie

Wasser aus dem Steinbruch in St. Magdalena bei Bozen.

Nach Auffindung der radioaktiven Quelle beim Schreiberhof in Tscherms zu Ende des Jahres 1911 erschien es der Mühe wert, das ganze Tonalitgebiet dieser Gegend nach Quellen abzusuchen, was nunmehr im Jahre 1912 auch durchgeführt wurde. Es fanden sich dabei auf der rechten Etschtalseite im Tonalitgebiet von Lana noch die etwas schwächer radioaktiven Quellen beim Rautnerhof in Tscherms, bei der Rateiser Mühle (diese aus aplitischem Granit) und beim Hofe Höllental (im Ultentale), welche sämtliche unmittelbar aus Fels entspringen, während die drei Trinkwasserquellen von Lana aus Schotter über Tonalit ihren Ursprung haben.

Auf der gegenüberliegenden Talseite im Tonalitgebiete des Ifingers weist nur die ebenfalls 1911 untersuchte Quelle am Kreuzjöchl bei Hafling (IV. Mitteilung) eine Radioaktivität 
von $8 \mathrm{M}$. E. auf; dieselbe entspringt nahe der Tonalitgrenze aus dem Grödner Sandstein. Dagegen sind die beiden Quellen in Gsteir (Naiftal), welche aus Trümmergestein (Blockhalden) an den Tag treten, fast ganz inaktiv. Beide Quellen fließen sehr wahrscheinlich längere Strecken mit starkem Gefälle unter den Trümmerhalden, so da $\beta$ ihre eigentliche Ursprungsstelle nicht zugänglich ist (beim Leiterbrunnen, einer sehr mächtigen Quelle, ist das unterirdische Rauschen des Wassers direkt vernehmlich). Es ist daher die Möglichkeit gegeben, daß die Radioaktivität dieser Wässer aus diesem Grunde nicht nachweisbar ist.

Bei den radioaktiven Quellen im Antholzertale (Bad Salomonsbrunn und Litzenbrunnen) ist deren außergewöhnliche Mächtigkeit besonders auffallend. Die Badequelle von Salomonsbrunn liefert nach Angabe des Besitzers 12 bis $15 l$ pro Sekunde und die Ergiebigkeit des Litzenbrunnens dürfte sich in ähnlichen Grenzen bewegen.

Das Produkt aus Mache-Einheiten und Sekundenlitern wäre dann eine Maßzahl für die Gesamtradioaktivität einer Quelle; dieselbe würde in beiden obigen Fällen ungefähr den Betrag 400 erreichen.

Herr Assistent Dr. R. Grengg hatte die Güte, die petrographische Untersuchung der Gesteine vom Schreiberhof in Tscherms bei Lana sowie von Bad Salomonsbrunn (Antholzertal) vorzunehmen und teilt darüber nachfolgendes mit:

\section{Granit von Tscherms bei Lana (Schreiberhof).}

Das ziemlich feinkörnige Gestein (mittlere Korngröße 2 bis $3 \mathrm{~mm}$ ) besteht aus vorwiegend Feldspat und Quarz nebst Biotit, der teilweise zersetzt ist; Schieferung ist weder im Handstück noch im Schliffe wahrnehmbar.

In den zur Untersuchung vorgelegten Proben fand sich auch ein grobkörniger Pegmatit, der den beschriebenen Granit durchsetzt und aus lichtrosa gefärbtem Kalifeldspat, Quarz und papierdünnen, großen Biotittafeln, die fast völlig zersetzt sind, besteht. Der Kalifeldspat des. Pegmatits ließ an einzelnen Spaltblättchen mikroperthitische Verwachsung mit Albit erkennen. 
Die Untersuchung des Granits im Dünnschliff ergänzt die gegebene Beschreibung in folgender Weise:

Orthoklas beteiligt sich in erster Linie an der Zusammensetzung des Gesteinsgewebes; er ist nicht mehr ganz frisch, die Durchschnitte erscheinen infolgedessen im durchfallenden Lichte (ohne Analysator) bräunlich getrübt durch kaolinartige Neubildungen; auch Muscovit, in feinen Schüppchen den Durchschnitten eingestreut, ist gleichfalls sekundär aus dem Feldspat entstanden. Der Orthoklas zeigt zuweilen Verzwillingung nach dem Karlsbader Gesetze sowie gewöhnlich mikropeothitische Durchwachsung mit Albit, welch letzterer auch vereinzelte Orthoklaskörner mantelartig umbüllt.

Der Plagioklas, nach dem Albitgesetze verzwillingt, ist von den beim Orthoklas beschriebenen Zersetzungsvorgängen noch stärker mitgenommen als dieser, und zwar sind es die Kernpartien der Körner, die umgewandelt sind, während die Hülle zumeist frisch blieb.

Während beim Kalifeldspat krystallographische Begrenzung der Durchschnitte so gut wie vollständig fehlt, tritt bei den Plagioklasen Neigung $z \mathfrak{u}$ idiomorpher Begrenzung deutlich ab und zu auf. Die geringe Höhe der Lichtbrechung, die Auslöschungsschiefe von $+14 \div 16^{\circ}$ in Schnitten $\perp M$ und $P$, ließen den vorliegenden Kalknatronfeldspat als zum AlbitOligoklas gehörig bestimmen. Bei einigen Plagioklasdurchschnitten war deutliche Zonenstruktur vorhanden; nach der höheren Lichtbrechung und der geringen Auslöschung $z \mathrm{u}$ schließen, ist die Hülle etwas anorthitreicher als der Kern, ein Verhältnis, wie es bei den Plagioklasen in Gesteinen aus der Reihe der krystallinischen Schiefer vorkommt.

Der Quarz zeigt keinerlei kataklastische Erscheinungen, die Durchschnitte sehen wie zart bestäubt aus infolge von Einschlüssen. Wegen ihrer Kleinheit konnten dieselben nicht näher bestimmt werden, nur vereinzelt ließ sich ein Flüssigkeitseinschluß mit Libelle eben noch wahrnehmen.

Biotit: Starker Pleochroismus in den gewöhnlichen Nuancen, beinahe optische Einachsigkeit, nicht seltene Einschlüsse von Zirkon und auch Apatit sowie opaken Körnchen, endlich die Umwandlung in grüne chloritische Sub- 
$\operatorname{stan} z$ charakterisieren diesen Bestandteil. Um die Zirkone finden sich $\mathrm{ab}$ und zu Zonen größerer Farbenintensität des Biotits (pleochroitische Höfe), deren Auftreten mit radioaktiven Wirkungen des Zirkons auf den Biotit in Verbindung gebracht wird.

\section{Gneis aus dem Antholzertal (Bad Salomonsbrunn).}

Im Handstück ist das Gestein deutlich geschiefert, auf günstigen Bruchflächen zeigt sich undeutlich grobflaserige Textur (Dimensionen der Flasern im Durchschnitte $7 \times 20 \mathrm{~mm}$ ). Die Korngröße ist sehr variabel, größere Körner von Feldspat (Augen) erreichen Dimensionen bis $1 \mathrm{~cm}$. Der Mineralbestand ist: Feldspat, Quarz, Glimmer (Muscovit und Biotit). Die Glimmerminerale sind um die einzelnen Flasern lagenweise angereichert, der Biotit ist zum Teil ausgebleicht und chloritisiert, sonst ist das Gestein frisch; auf Kluftflächen finden sich öfters dünne Überzüge von Eisenoxyd. An einem der vorgelegten Stücke war die von Flechten teilweise bedeckte Oberfläche auf mehreren Millimetern Tiefe in lichtgelbrot gefärbten sandigen Grus mit Glimmerschüppchen verwittert.

Die Untersuchung im Dünnschliff gibt noch folgende Details: Am Gesteinsgewebe beteiligen sich vorwiegend Kalifeldspat, und zwar Mikroperthit und Mikroklin, dann Plagioklas, Quarz, die beiden Glimmer, wobei der Muscovit öfters in Serizitaggregate sich ausfasert, während der Biotit Entfärbung sowie Umwandlung in Chlorit zeigt. $\mathrm{Ab}$ und $\mathrm{zu}$ eingestreut fanden sich kleinere Durchschnitte eines sehr schwach lichtbrechenden, optisch isotropen und gut spaltbaren, krystallographische Begrenzung nicht zeigenden Minerals, das mit Fluorit ident zu sein scheint.

Der Kalifeldspat ist ab und zu verzwillingt, Zersetzungserscheinungen fehlen so ziemlich, desgleichen beim Mikroklin, der gleichfalls von Albit durchwachsen ist, und bei den Plagioklasen, die sich als zum Albit-Oligoklas (Auslöschungsschiefe auf Schnitten $\perp M$ und $P=+16^{\circ}$ ) gehörig bestimmen ließen. Am Quar $z$, der voll staubartig kleiner Einschlüsse ist, und am Plagioklas, wie am Glimmer zeigt sich an Verbiegungen und optischen Störungen mechanische Beanspruchung des Gesteins. 
Besonders in den Plagioklasschnitten finden sich öfter massenhaft Einschlüsse von zumeist Muskovit; diese Schüppchen sind idiomorph und als primäre Bildungen anzusehen.

Sonst im Schliff überwiegt der Muscovit gegen den Biotit, letzterer zeigt ab und zu pleochroitische Höfe wohl um winzige Zirkone, diese Höfe sind auch dort als scharfe Pupillen um den Einschluß zu sehen, wo der Glimmer in grüne Chloritsubstanz übergegangen ist. Opake Partikeln in und um die Glimmer sind spärlich vorhanden.

Den kommunalen Verwaltungen sowie den Besitzern und Direktoren der verschiedenen Bäder und Kuranstalten danken wir verbindlichst für das freundli the Entgegenkommen, das sie uns bei Ausführung dieser Arbeiten angedeihen ließen.

$\mathrm{Zu}$ besonderem Danke sind wir Fräulein M. Pimmer sowie Herrn Assistenten Dr. R. Grengg und Ilemin Prof. J. Andreatta verpflichtet. 